\title{
Big Science e a formação do modelo de partículas elementares
}

\author{
R.C. Nunes ${ }^{1}$; J.M. Dantas ${ }^{1,2}$; J.E. Andrade ${ }^{1,3}$ \\ ${ }^{I}$ Mestrado Profissional em Ensino de Física/Instituto de Ciências Exatas, Universidade Federal do Sul e Sudeste do \\ Pará, 68500-000, Marabá-PA \\ ${ }^{2}$ Faculdade de Computação e Engenharia Elétrica/Instituto de Geociências e Engenharias, Universidade Federal do \\ Sul e Sudeste do Pará, 68507-590, Marabá - Pará, Brasil \\ ${ }^{3}$ Faculdade de Engenharia Mecânica/Instituto de Geociências e Engenharias, Universidade Federal do Sul e Sudeste \\ do Pará, 68507-590, Marabá - Pará, Brasil \\ robertocostanunes@yahoo.com.br
}

(Recebido em 26 de setembro de 2016; aceito em 27 de dezembro de 2016)

\begin{abstract}
Resumo: O presente trabalho procura discursar um pouco acerca do modelo de partículas elementares com base no Modelo Padrão da física de partículas. Para isso será versado sobre o termo Big Science e ainda um breve processo histórico da formação do modelo de partículas elementares. Diante disso, será exposto o modelo discutido atualmente das partículas elementares, concluindo a imensa capacidade da produção de conhecimento, que leva ao entendimento do comportamento da natureza.
\end{abstract}

Palavras-chave: Big Science, Física de Partículas, Modelo de partículas elementares.

Abstract: This paper seeks to address the model of elementary particles based on the Standard Model of particle physics. To this will be versed on the term Big Science and also a brief historical process of formation of elementary particle model. Therefore, it will be exposed a model currently discussed of elementary particles, completing the immense capacity of knowledge production, which leads to the understanding of the behavior of nature.

Keywords: Big Science, Particle Physics, Model of elementary particles.

\section{INTRODUÇÃO}

$\mathrm{O}$ estudo e pesquisa em Física tem proporcionado constante crescimento na produção de conhecimento no que tange a compreensão da natureza, envolvendo o comportamento mais íntimo dos fenômenos naturais. E nos últimos cem anos, uma parte da Física tem experimentado descobertas fantásticas, proporcionando o entendimento elementar da matéria que forma tudo que é conhecido: Física de partículas [1].

Fazer ciência é um termo associado ou até mesmo sinônimo de pesquisa científica, e certamente a pesquisa realizada nos tempos atuais é bem diferente do que era feito no passado. Para a Física de partículas é essencial o investimento financeiro e humano, necessitando de altos recursos financeiros. A importância para a pesquisa é tão notória que é observável o esforço para captação desses recursos, seja pelo Estado, seja por empresas (ou união de empresas), para procurar cada vez mais novas descobertas. Assim, é associado à pesquisa o surgimento de novas tecnologias. Fruto de tanto esforço pode ser observado diretamente ou indiretamente, em quase tudo que o homem pode usar.

Para falar acerca da história das partículas elementares, é necessário que se fale também sobre o termo Big Science, uma vez que esse se trata basicamente do investimento massivo nas pesquisas científicas.

\section{BIG SCIENCE}

O termo Big Science foi cunhado por Alvin M. Weinberg, em 1961, então diretor do laboratório Nacional de Oak Ridge, na revista Science, com o seu trabalho intitulado Impact of Large-Scale Science on the United States. Nesse trabalho, é colocada a questão do investimento em larga escala em ciência, principalmente durante e após a Segunda Guerra Mundial, onde a 
construção do conhecimento científico estava mais voltada para a questão militar, tendo sido obtidos importantes avanços na física, eletrônica, microeletrônica, aeronáutica, bélica, nuclear, espacial entre outras áreas [2].

Dessa forma, o termo Big Science está ligado com o investimento em larga escala nas pesquisas científicas. Isso leva a mudanças na forma de como a ciência é feita, além da concepção de como o conhecimento científico é construído, alterando até mesmo a sua finalidade. Antigamente as descobertas em ciência eram atribuídas à genialidade de uma pessoa, onde em grande parte esse esforço era individual ou com pouca colaboração de terceiros, e geralmente por curiosidade do próprio pesquisador em querer explicar o comportamento da natureza. Já na era do Big Science, as descobertas são alcançadas por grupos de pesquisadores que trabalham em conjunto, buscando além da explicação do comportamento da natureza, a solução de problemas.

O questionamento enfatizado na era do Big Science é sobre a mudança da característica fundamental da pesquisa, pois tais pesquisas estariam mais voltadas ao desenvolvimento de novos produtos. Assim, vale a pena um pequeno e breve diálogo sobre o assunto. Ciência de verdade está envolvida com descobertas, e isso leva ao aprimoramento do conhecimento entendendo como as coisas funcionam e se comportam na natureza. Pesquisas que envolvem invenções também têm a sua importância para a sociedade, todavia nem sempre carregam a essência da pesquisa científica que é nas predições: comprovação das teorias. Invenções não são descobertas, mas uma descoberta pode proporcionar novas invenções.

Outro ponto interessante que pode ser levantando com investimento em ciências, é buscar justamente pelas comprovações de teorias científicas. Podemos perceber no passado que as teorias e leis eram formuladas com a observação de determinado comportamento da natureza, explicando tais comportamentos de forma categórica e condizente com o método científico. Só que quando as coisas não podem ser tão bem observadas, as teorias são formuladas com base em modelos bem fundados, porém a sua observação e comprovação necessita de grandes recursos. Exemplos atuais seriam justamente o bóson de Higgs e as ondas gravitacionais, onde tais fenômenos foram poderosas predições formuladas com o conhecimento da Física, predições tão fortes que levaram ao investimento de altos valores para a construção de equipamentos que puderam comprovar tais teorias.

Assim, com grandes investimentos surgiram centros de pesquisas e laboratórios gigantescos puderam ser formados. Para a Física de partículas, o maior ganho veio pelo surgimento dos aceleradores de partículas construídos pelos centros de pesquisas. Talvez, dentre todos esses centros, o mais conhecido deve ser o CERN, antigo acrônimo para Conseil Européen pour la Recherche Nucléaire, hoje chamada de Organização Europeia para Pesquisa Nuclear.

Com mais de 60 anos de história e formada por 21 países membros, o CERN é na verdade uma organização, representada por centenas de universidades e centros de pesquisas, possuindo milhares de cientistas e engenheiros, sendo o detentor do maior acelerador de partículas, conhecido como LHC (Large Hadron Collider, ou Grande Colisor de Hádrons). O LHC é um túnel subterrâneo circular de $27 \mathrm{~km}$ de circunferência e que fica pouco mais de 100 metros abaixo do nível do solo, custando algo em torno de 8 bilhões de dólares.

\section{O SURGIMENTO DA FÍSICA DE PARTÍCULAS}

Os segredos do gigantesco universo estão justamente na compreensão das minúsculas partículas que formam toda a matéria conhecida, e a busca pela elementaridade da matéria não é algo restrito aos tempos modernos. Já no século IV a.C., Demócrito e Leucipo já tentavam explicar que toda a matéria conhecida deveria ser formada por pequenas partículas indivisíveis, chamadas de átomos. Porém, essa ideia acabou ficando hibernada por mais de 25 séculos, prevalecendo em maior parte o pensamento de que tudo deveria ser formado pela união dos quatro elementos da natureza: terra, água, ar e fogo [1].

Jhon Dalton voltou a defender a ideia dos átomos como a menor parte da matéria, porém coube a Joseph Jhon Thomson demonstrar que até mesmo o átomo pode ser dividido, sendo formado por partículas ainda menores. Ernest Rutherford e Niels Borhs contribuíram ainda mais para a construção do modelo atômico formado por prótons, nêutrons e elétrons. 
Desde a comprovação e aceitação dos átomos do início do século XIX, o conceito de partícula elementar passou por diversas transformações, e o modelo de partículas elementares sofreu constantes mudanças. Na Física Moderna, o conceito de partículas elementares levou a divisão de partículas a outro nível. Com a descoberta do elétron por Joseph Jhon Thomson, foi então iniciada a busca pelas partículas elementares, onde até mesmo um próton ou nêutron pode ser dividido em partículas ainda menores [1].

Percebe-se aí a formação do conhecimento realmente de forma construtiva, onde ideias formuladas, às vezes podem estar ligeiramente equivocadas, e podem ser melhoradas. Tanto esforço requer também recursos, e assim juntamente com o trabalho intelectual humano, é necessário ter-se aparato tecnológico para proporcionar tais descobertas.

\subsection{MODELO DE PARTÍCULAS ELEMENTARES}

A Física de Partículas procura explicar a natureza da matéria em sua forma mais essencial possível. Com isso, há o Modelo Padrão das partículas elementares, que não é propriamente dito um modelo, e sim uma teoria, a qual busca identificar as partículas básicas e especificar como elas interagem $[3,4]$.

Até onde se sabe, de acordo com o Modelo Padrão, há as partículas verdadeiramente elementares, pois elas não possuem estrutura interna e não são divisíveis ainda podendo ser léptons ou quarks. Na categoria léptons, há o elétron, múon, tau, neutrino do elétron, neutrino do múon e neutrino do tau. Quanto aos quarks, há o quark up, quark down, quark charme, quark estranho, quark bottom e quark top. Só que, os quarks possuem uma propriedade conhecida como cor, ou melhor, carga cor (vermelho, verde e azul). Assim, cada um dos seis quarks pode existir de três formas diferentes. Sendo assim, há os 6 léptons e 18 quarks, e como para cada partícula existe uma antipartícula correspondente, pode-se dizer que existem 12 léptons e 36 quarks [3].

As partículas que possuem estrutura interna são chamadas de hádrons, podendo ser bárions (formadas por 3 quarks ou 3 antiquarks) ou mésons (formadas por 1 quark e 1 antiquark). Certamente o elétron é o léptons mais versado, enquanto o próton e o nêutron são os exemplos de hádrons popularmente conhecidos.

Sabe-se que prótons, elétrons e nêutrons possuem uma determinada carga elétrica de $+1 e,-1 e$ e 0 , respectivamente. Tanto prótons quanto nêutrons são bárions, onde o primeiro é formado por dois quarks up e um quark down, enquanto o segundo é formado por um quark up e dois quarks down. Os quarks possuem uma carga elétrica fracionária, de modo que no próton, a soma das cargas elétricas individuais de seus quarks dê $+1 e$, enquanto que no nêutron essa resultante será nula. Veja na tabela 1 que cada tipo de quark possui uma fração da carga elementar.

Tabela 1: Algumas características dos quarks

\begin{tabular}{l|c|c}
\hline Nome (símbolo) & Carga elétrica & Carga cor \\
\hline Up (u) & $+\frac{2}{3} e$ & $\mathrm{R}, \mathrm{G}, \mathrm{B}$ \\
\hline Down (d) & $-\frac{1}{3} e$ & $\mathrm{R}, \mathrm{G}, \mathrm{B}$ \\
\hline Strange (s) & $-\frac{1}{3} e$ & $\mathrm{R}, \mathrm{G}, \mathrm{B}$ \\
\hline Charm (c) & $+\frac{2}{3} e$ & $\mathrm{R}, \mathrm{G}, \mathrm{B}$ \\
\hline Bottom (b) & $-\frac{1}{3} e$ & $\mathrm{R}, \mathrm{G}, \mathrm{B}$ \\
\hline Top (t) & $+\frac{2}{3} e$ & $\mathrm{R}, \mathrm{G}, \mathrm{B}$ \\
\hline
\end{tabular}

No caso do próton (com estrutura interna uud), ao somar o valor da carga de dois quarks up $(+2 / 3 e)$ com um quark down $(-1 / 3 e)$, será possível observar que a resultante de suas cargas será de $+1 e$, condizente com a carga conhecida do próton. O mesmo vale para o nêutron (com 
estrutura interna udd), onde a resultante da soma das cargas elétricas de um quark up e dois quark down (consulte a tabela acima), será zero [3,5].

Dentro do Modelo Padrão pode-se ter uma grande diversidade de partículas, sendo que elas podem ser verdadeiramente elementares, ou podem ser formadas por uma combinação dessas tendo uma estrutura interna. Vamos organizar essas partículas no fluxograma abaixo.

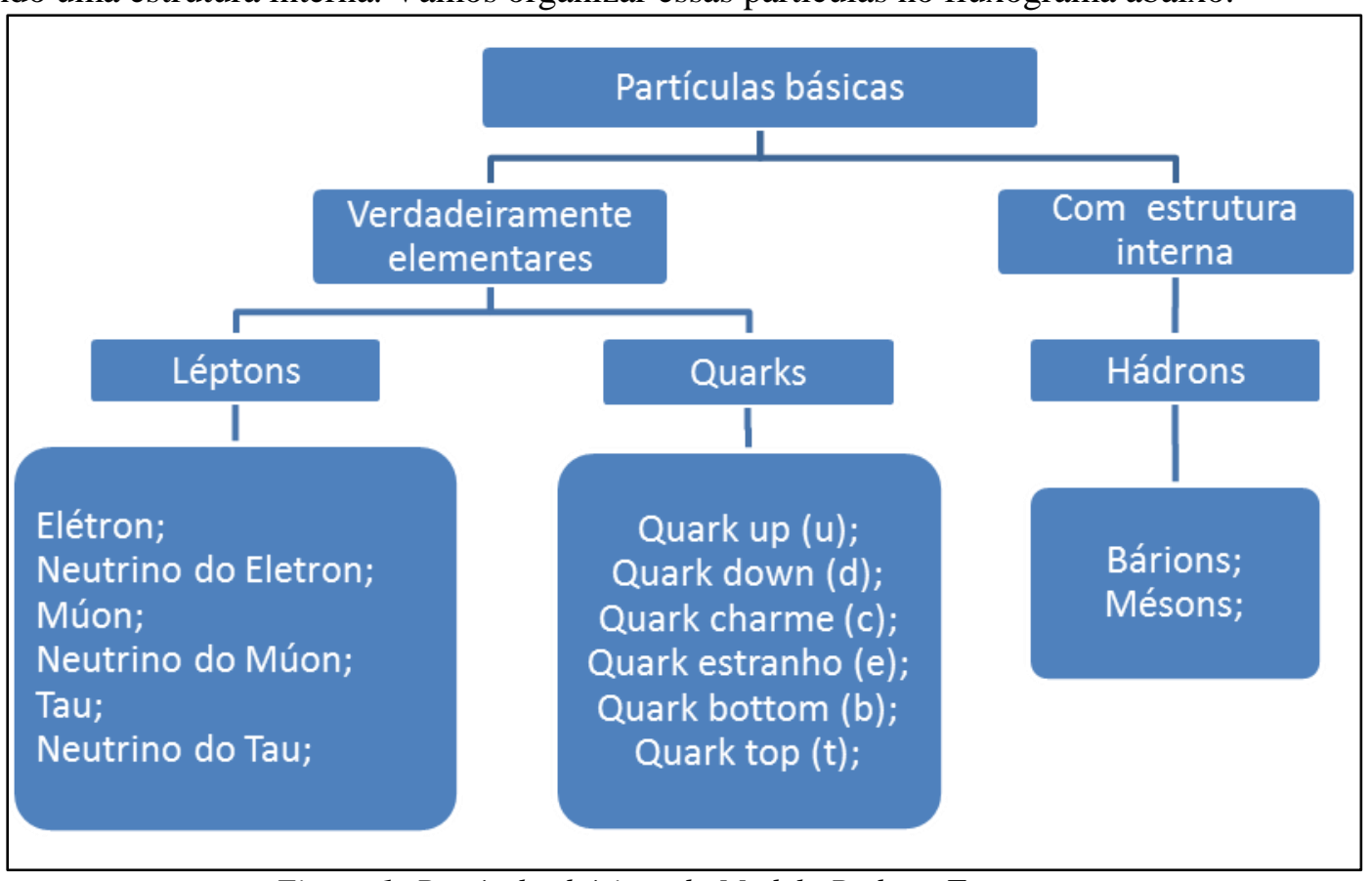

Figura 1: Partículas básicas do Modelo Padrão. Fonte: autor.

Um ponto curioso no Modelo Padrão, é que nada impede dos quarks existirem sozinhos, mas eles nunca foram observados livres, sempre confinados em hádrons. Aparentemente tendem a manter o quantum da carga elétrica, como exemplo o próton, ou uma carga elétrica nula, por exemplo o nêuton.

Isso é a base do modelo padrão, mas não é ele por completo. Primeiro, por que novas partículas ainda podem ser descobertas, podendo levar adaptações ou mudanças desse modelo. Segundo, que falta especificar as interações dessas partículas.

De acordo com Griffths [6], existem quatro forças fundamentais conhecidas atualmente na física, valendo ressaltar que são a força forte, força eletromagnética, força fraca e força gravitacional. Essas forças aparecem devido às quatro interações fundamentais, sendo que cada uma das interações é devida a uma propriedade fundamental da matéria. A interação forte é devida a carga cor, interação eletromagnética à carga elétrica, interação fraca à carga fraca e interação gravitacional à massa $[3,5]$.

Forças como a elástica, atrito, contato e intermoleculares são apenas exemplos de forças eletromagnéticas. A força gravitacional possui uma intensidade tão baixa que só pode ser percebida em corpos com massa bastante elevada. Em ordem de intensidade, essas quatro forças podem ser listadas da seguinte forma: Forte > Eletromagnética > Fraca > Gravitacional [6].

As forças existem pelas interações, e as interações vão ocorrer como resultado da troca de partícula, entrando em jogo as partículas mediadoras. Cada tipo de interação vai ocorrer pela troca de certo tipo de partícula mediadora específica. No caso da interação forte haverá a troca de glúons, na interação eletromagnética a troca de fótons, na interação fraca as partículas W e Z, e na gravitacional os grávitons.

Falta ainda falar dos campos de atuação de cada uma dessas interações. A interação gravitacional gera um campo gravitacional, a interação eletromagnética gera um campo eletromagnético, a interação forte um campo forte, enquanto a interação fraca um campo fraco. $O$ campo gravitacional e o eletromagnético possui um alcance extenso, enquanto os campos fortes e fracos têm ação em pequenas distâncias. Essas partículas mediadoras são chamadas de partículas virtuais por serem pulsos de energia, onde o fóton e o glúon não possuem massa, enquanto as 
partículas $\mathrm{W}$ e $\mathrm{Z}$ possuem. Mesmo as partículas $\mathrm{W}$ e $\mathrm{Z}$ tendo massa, elas são consideradas virtuais por se manifestarem como mediadora [4].

Sintetizando as interações:

- Eletromagnética: devido à carga elétrica das partículas que estão interagindo, criando um campo eletromagnético (lembrando que quando a carga está em repouso é percebido apenas o campo elétrico, e quando em movimento também o campo magnético), sendo que exerce a força eletromagnética e é transmitida pelos fótons;

- Forte: essa interação é devida a carga cor, criando o campo forte de alta intensidade e baixo alcance, exercendo a força forte e é transmitido pelos glúons;

- Fraca: devido a instabilidade da partícula, que leva à transmutação (decaimento) da partícula, tendo o campo fraco, sendo que exerce a força fraca e é transmitida pelas partículas W e Z.

- Gravitacional: devido à massa, e cria o campo gravitacional, o qual possui grande alcance, porém de baixa intensidade, tendo exercida a força gravitacional e é transmitida pelas partículas mediadoras grávitons;

\section{CONCLUSÃO}

Por que tanto esforço para descobrir os detalhes do universo? Certamente a resposta mais óbvia é que seria justamente nos detalhes em que se encontram os segredos para a compreensão da matéria. A natureza possui diversas formas de comportamento, mas nisso tudo há padrões. Assim como a percepção do padrão de dia leva o entendimento de tempo passando, a percepção da elementaridade pode levar a compreensão do padrão das múltiplas formas de manifestação da natureza. $\mathrm{O}$ que isso proporciona? Tanto investimento de tecnologia requer também conhecimento, tanto para compreender o que se faz e o que se busca, como para manusear tecnologia e dados, e isso resulta em mais conhecimento. Tal ciclo leva ao entendimento do que acontece ao nosso redor, ou pelo menos de parte disso.

Para a física de partículas pode-se perceber a descoberta de certos padrões das partículas elementares, o que já é por si só foco de bastante estudo. Mas sabe-se também que isso ainda não é suficiente, tendo ainda muitas outras novas coisas para serem descobertas. Mesmo sabendo como parte da matéria é formada e como ela se comporta, é sensato afirmar que há muito mais coisas que não sabemos como são formadas e por que se comportam de tal maneira.

Dessa forma, a pesquisa sobre as partículas elementares deve ser continuada e os resultados já obtidos, que é o conhecimento atual sobre o assunto, devem ser discutidos de forma mais ampla, disseminando assim esse conhecimento tão valioso.

\section{REFERÊNCIAS BIBLIOGRÁFICAS}

1. ABDALlA, Maria Cristina Batoni. O Discreto Charme das Partículas Elementares. Física na Escola, v.6, n.1, p. 38-44. 2005.

2. WEINBERG, Alvin M. Impact of Large-Scale Science on the United States. Science, Tennessee, v. 134, n. 3473, p.161-164, 21 jul. 1961.

3. MOREIRA, Marco Antonio. Partículas e Interações. Física na Escola, v.5, n.2, p. 10-14. 2004.

4. MOREIRA, Marco Antonio. O Modelo Padrão da Física de Partículas. Revista Brasileira de Ensino de Física, Porto Alegre/RS, v. 31, n. 1, p.1306-1 - 1306-11, 30 abr. 2009.

5. OSTERMANN, F. e CAVALCANTI, C.J.H. (2001). Um pôster para ensinar Física de Partículas na escola. Física na Escola. v.2, n.1, p.13-18. 2001.

6. Griffiths, David J. Eletrodinâmica. São Paulo: Pearson, 3 ed. p.13. 